\title{
Reduced-Dimension Power Allocation over Clustered Channels in Cognitive Radios System under Co-channel Interference
}

\author{
Mahdi Ben Ghorbel*, Mamoun Guenach\#, Mohamed-Slim Alouini* \\ ${ }^{*}$ King Abdullah University of Science and Technology (KAUST), Thuwal, Makkah Province, Saudi Arabia \\ Email: \{mahdi.benghorbel, slim.alouini\}@kaust.edu.sa \\ \#Email: guenach@ieee.org
}

\begin{abstract}
The objective of this paper is to propose a reduceddimension resource allocation scheme in the context of cognitive radio system in presence of co-channel interference between users. We assume a multicarrier transmission for both the primary and secondary systems. Instead of optimizing the powers over all sub-carriers, the sub-carriers are grouped into clusters of sub-carriers, where the power of each sub-carrier is directly related to the power of the correspondent cluster. The power optimization is done only over the set of clusters instead of all sub-carriers which can significantly reduce the complexity of the resource allocation problem. The performance loss of the reduced dimension solution with respect to the optimal solution, where the optimization is carried over all active sub-carriers, allows trading-off complexity versus performance. Numerical evaluation indeed revealed that a limited performance loss occurs by optimizing over a reduced set of clusters instead of the full optimization in the context of cognitive radio systems.
\end{abstract}

\section{INTRODUCTION}

The concept of cognitive radio was first presented by Mitola [1] to solve the problem of spectrum scarcity pointed by the Federal Communication Commission report [2]. The main principle of this concept is to let secondary (cognitive) users access the spectrum of the primary users opportunistically under the condition of satisfying e.g. an interference constraint to the primary user. There are three main paradigms for cognitive radio as classified by Goldsmith et al. [3]: 1) Interweave systems, in which cognitive users are allowed to transmit only in the channels where the primary user is absent; 2) in underlay systems, the secondary users are allowed to use all channels but under the constraint that the total interference caused to the primary user does not exceed a threshold level while in 3) overlay systems, secondary users will help the primary user improving its performance by playing the role of relays, and at the same time take advantage from the knowledge of the primary user code-books to transmit in parallel for its own data without harming the primary user. In our work, we consider a hybrid scheme of interweave and underlay. It consists first in sensing the activity of the primary user and then transmitting without limit when it is absent (like the interweave case), and under the interference constraint when it is present (like the underlay case). This hybrid scheme allows a better exploitation of the available channels for transmission. The two original schemes can be deduced from this scheme by simple choice of the interference parameters.

The dynamic power allocation in the context of cognitive radios has been extensively studied in the context of cognitive radio systems [4], [5], [6]. Due to the nature of cognitive radio requiring rapid exploitation of the opportunities that occur before the status of the primary users change, complexity reduction of the resource allocation algorithms remains a challenge for this system. In multicarrier systems like Orthogonal Frequency-Division Multiple Access (OFDMA), the selection of the sub-carrier bandwidth is a key factor since it controls the trade-off between computational complexity and performance. For instance, a large sub-carrier width allows to reduce notably the computational complexity under the assumption of constant channel gains over each sub-carrier which is not usually the case in wireless channels. On the other hand, reducing the sub-carrier width results in large number of channels that have to be optimally allocated; Hence the computational complexity of the resource allocation problem becomes cumbersome, which is not desirable in rapidly changing channels.

In this work, we propose a low complexity algorithm for the channel assignment under co-channel interference. In the proposed scheme the available sub-carriers are grouped into clusters or blocks of sub-carriers where the size of each cluster is a design parameter that depends on e.g. the spectral properties of the channels. Thus, the power allocation will be performed over reduced number of clusters instead of all sub-carriers. An interpolation matrix will be defined to relate the power allocation per sub-carrier and the relevant cluster's power. The regrouping of the sub-carriers will depend on the channel and noise smoothness. The choice of the clustering is not the focus of this paper; we rather work with predefined clusters and concentrate on how to solve the reduced dimension optimization problem and evaluate its performance and complexity compared to the optimal scheme where the optimization is carried over all sub-channels. We should note that similar ideas of reduced dimension spectrum allocation were studied in the context of digital subscriber line systems [7]. However, the problem here is treated with different interpolation matrix in addition to the additional challenges of the wireless channels and the cognitive radio 
context.

We will propose a resource allocation problem were the weighted sum of the achievable rates of the different users is maximized in the utility function, under power and target rate constraints in addition to the interference constraint to the primary users. We will formulate the optimization problem considering the clustering of sub-carriers and show that the new problem with clustering can still be decomposed into per cluster independent sub-problems. The complexity gain using this approach is then proportional to the average number of sub-carriers per cluster. Inherent to this interpolation, there is a performance loss that will be evaluated by simulations.

The rest of the paper is organized as follows: We describe in section II the cognitive system model and formulate the resource allocation optimization problem under the clustering property in section III. In section IV, we analyze and solve the optimization problem. In section $\mathrm{V}$, we present some numerical simulation results showing the complexity/performance tradeoffs of the reduced dimension approach. Finally, in section VI we draw some conclusions.

\section{SyStem Model}

Consider a cognitive radio system composed of $K_{c}$ secondary users and $K_{p}$ primary users sharing $N$ sub-carriers in an OFDMA based transmission. We denote by $H_{p_{l}, c_{u}}^{(n)}$ the channel gain from the cognitive user $u$ to the primary user $l$, and $H_{c_{k}, c_{u}}^{(n)}$ the channel gain from the cognitive user $u$ to the cognitive user $k$. The channel gains from the secondary to the primary users are assumed to be known by the secondary users.

\section{Problem Formulation}

We consider as a utility function the maximization of the weighted sum of the cognitive users' rates

$$
\max _{P_{u}^{(n)}} \sum_{u=1}^{K_{c}} \alpha_{u} R_{u}
$$

where

- $\alpha_{u}$ is the weight associated to the rate of the user $u$,

- $P_{u}=\sum_{n=1}^{N} P_{u}^{(n)}$ is the total power of the user number $u$ that cannot exceed certain maximum budget denoted $\hat{P}_{u}$,

- $P_{u}^{(n)}$ is the power allocated to the user $u$ on the subcarrier $n$ that is upper bounded by a per-subcarrier mask denoted $\hat{P}_{u}^{(n)}$,

- $R_{u}=f_{s} \sum_{n=1}^{N} \log _{2}\left(1+\frac{\gamma_{u}^{(n)}}{\Gamma_{u}}\right)$ refers to the user $u$ rate, with $f_{s}$ the symbol rate in $\mathrm{Hz}, \Gamma_{u}$ the total gap to capacity containing the effects of the coding gain and the discrete bit loading, and $\gamma_{u}^{(n)}$ the signal-to-interference plus noise ratio (SINR) of the user $u$ over the $n$-th sub-carrier expressed as

$$
\gamma_{u}^{(n)}=\frac{\left|H_{c_{u}, c_{u}}^{(n)}\right|^{2} P_{u}^{(n)}}{\sum_{\substack{k=1 \\ k \neq u}}^{K_{c}}\left|H_{c_{u}, c_{k}}^{(n)}\right|^{2} P_{k}^{(n)}+N_{u}^{(n)}},
$$

where $N_{u}^{(n)} / f_{s}$ is the power spectral density of the background noise which incorporate implicitly interference from primary users or other devices as well.

\section{A. Interference Constraint in Cognitive Radio Systems}

In cognitive radio systems, the interference constraint is a key parameter as it directly affects the achievable performance of the cognitive system as well as the protection level of the primary users against the interference from the secondary users. Although different formulations where proposed in literature in order to reduce the primary user's feedback or channels estimation overhead, in this work we consider a standard instantaneous interference constraint as follows:

$$
B_{l}^{(n)} \sum_{u=1}^{K_{c}}\left|H_{p_{l}, c_{u}}^{(n)}\right|^{2} P_{u}^{(n)} \leq \check{I}_{l}^{(n)} \forall l, n .
$$

This constraint ensures that for each sub-carrier $n$ where the primary user $l$ is active, the total power received from the different cognitive users should not exceed a fixed interference threshold $\check{I}_{l}^{(n)}$. The activity of the primary user is defined by the $N \times K_{p}$ indexing matrix $\mathbf{B}\left(B_{n, l}=B_{l}^{(n)}\right)$, where $B_{l}^{(n)}=1$ indicates that the $l$-th primary user is active on the $n$-th sub-carrier and $B_{l}^{(n)}=0$ otherwise. The advantage of this interference constraint is that it ensures instantaneous protection to the primary users but it assumes an instantaneous estimation of the interference channel to the primary users and feedback about the tolerable interference per sub-carrier. The interference threshold $\check{I}_{l}^{(n)}$ can be determined in function of the required bit loading or the tolerable SINR of the primary user. This interference threshold is assumed to be known by the cognitive system in this paper.

\section{B. Sub-carriers Clustering}

The sub-carriers are grouped into $M \leq N$ clusters. An $N \times M$ binary indicator matrix $\mathbf{A}$ relates the active sub-carriers to the different clusters where $A_{n, m}=1$ means that subcarrier $n$ belongs to cluster $m$ and otherwise $A_{n, m}=0$. In the present clustering formulation, we consider the case when each sub-carrier can only belong to one cluster; hence we have per row of the indicator matrix $\mathbf{A}$ the following constraint $\sum_{m=1}^{M} A_{n, m}=1, \forall n$. Thus, the power allocation for each sub-carrier can be deduced from the power allocated to each cluster as

$$
P_{u}^{(n)}=\sum_{m=1}^{M} A_{n, m} \cdot Q_{u}^{(m)}=A_{n, m_{n}} \cdot Q_{u}^{\left(m_{n}\right)}
$$


where $P_{u}^{(n)}$ is the power spectrum assigned to the sub-carrier $n$ of user $u, Q_{u}^{(m)}$ is the power assigned to sub-carriers belonging to the cluster $m$ of the user $u$. The index $m_{n}$ of the cluster to which the sub-carrier $n$ belongs is defined as

$$
\forall n, \exists ! m_{n}, A_{n, m}= \begin{cases}1 & \text { if } m=m_{n} \\ 0 & \text { otherwise. }\end{cases}
$$

This indexing matrix can be generalized to an interpolation matrix where the parameters $A_{n, m}$ can take not only binary values 0 or 1 but any positive real value in the interval $[0,1]$. In this case, the parameters $A_{n, m}$ will play the role of scaling coefficients of the sub-carrier power in reference to the subgroup power to which they belong. The only constraint in this generalized case is that a sub-carrier should belong to only one cluster, i. e. $0<A_{n, m_{n}} \leq 1$ and $A_{n, m \neq m_{n}}=0$. Inversely, the power per cluster can be obtained from the subcarrier powers as

$$
Q_{u}^{\left(m_{n}\right)}=\frac{P_{u}^{(n)}}{A_{n, m_{n}}}, \forall n
$$

The scaling coefficients $A_{n, m_{n}}$ can be determined using e.g. the shape of the mask and empirical models of the channel dependency versus frequency. The choice of such coefficients is beyond the scope of this paper. We rather focus on a indexing interpolation where $A_{n, m_{n}}=1$ and $A_{n, m \neq m_{n}}=0$. We assume that the clustering is selected according to the primary users activity in order to ensure that the primary users have the same state for all the sub-carriers of the same cluster (either active or inactive over all sub-carriers in each cluster). Thus, for each cluster $m$ and all sub-carriers $n$ belonging to it, we have $B_{l}^{(n)}=B_{l}^{\left(m_{n}\right)}, \forall l$. This assumption is realistic since in practice usually spectrum allocation is done similarly over adjacent sub-carriers. For example in Long-Term Evolution (LTE), Orthogonal Frequency-Division Multiple Access is used with blocks of 12 sub-carriers. Thus, the primary occupation will be the same for all sub-carriers in each block.

\section{Reduced Dimension Power Allocation}

\section{A. Optimization Problem}

We consider a centralized scheme where a central unit (for example the base station in cellular system) will collect all users' requirements and channel states to distribute the resources in an optimal way. Taking the utility function in (1) under the constraints of 1) maximum power budget per user $\left.\hat{P}_{u}, 2\right)$ maximum power per user per sub-carrier $\hat{P}_{u}^{(n)}$, and 3) maximum interference to each primary user $l, \check{I}_{l}^{(n)}$ as described in (3). The optimization problem is then formulated as

$$
\begin{array}{ll} 
& \max _{P_{u}^{(n)}} \sum_{u=1}^{K_{c}} \alpha_{u} R_{u} \\
\text { S.t } & P_{u} \leq \hat{P}_{u} \forall u \\
& 0 \leq P_{u}^{(n)} \leq \hat{P}_{u}^{(n)} \forall u, \forall n \\
& B_{l}^{(n)} \sum_{u=1}^{K_{c}}\left|H_{p_{l}, c_{u}}^{(n)}\right|^{2} P_{u}^{(n)} \leq \check{I}_{l}^{(n)} \forall l, \forall n .
\end{array}
$$

Using the clustering characteristic expressed in (4), the rate of user $u$ can be rewritten as

$$
R_{u}=\sum_{m=1}^{M} \sum_{n=1}^{N} f_{s} \log _{2}\left(1+\frac{\gamma_{u}^{(m, n)}}{\Gamma_{u}}\right)
$$

with $\gamma_{u}^{(m, n)}$ defined as the SINR of the user $u$ over the $n$-th sub-carrier in cluster $m$ expressed as

$$
\gamma_{u}^{(m, n)}=\frac{A_{n, m}\left|H_{c_{u}, c_{u}}^{(n)}\right|^{2} Q_{u}^{(m)}}{\sum_{k=1, k \neq u}^{K_{c}} A_{n, m}\left|H_{c_{u}, c_{k}}^{(n)}\right|^{2} Q_{k}^{(m)}+N_{u}^{(n)}},
$$

where $Q_{k}^{(m)}$ is the power loading in the $m$-th cluster for the $k$-th user. We note that as postulated in the characterization of the clusters, for a sub-carrier $n$ that does not belong to a cluster $m\left(m \neq m_{n}\right)$, the interpolation index is null (i.e., $A_{n, m}=0$ ) leading to $\gamma_{u}^{(m, n)}=0$. Thus, in the expression of the rate in (7), only the terms corresponding to the sub-carriers belonging to each cluster $m$ will remain in the sum.

Following this clustering relations, the problem (6) can be reformulated as follows

$$
\begin{aligned}
& \max _{Q_{u}^{(m)}} \sum_{u=1}^{K_{c}} \alpha_{u} \sum_{m=1}^{M} \sum_{n=1, A_{n, m} \neq 0}^{N} f_{s} \log _{2}\left(1+\frac{\gamma_{u}^{(m, n)}}{\Gamma_{u}}\right) \\
& \text { S.t. } \sum_{m=1}^{M} \sum_{n=1}^{N} A_{n, m} Q_{u}^{(m)} \leq \hat{P}_{u} \forall u \\
& 0 \leq Q_{u}^{(m)} \leq \min _{\substack{1 \leq n \leq N \\
A_{n, m} \neq 0}}\left\{\frac{\hat{P}_{u}^{(n)}}{A_{n, m}}\right\} \forall u, \forall m \\
& A_{n, m} B_{l}^{(n)} \sum_{u=1}^{K_{c}}\left|H_{p_{l}, c_{u}}^{(n)}\right|^{2} Q_{u}^{(m)} \leq \check{I}_{l}^{(n)} \forall l, \forall(m, n) .
\end{aligned}
$$

Thus, the problem is shown to be re-rewritten in function of the new $K_{c} \times M$ optimization variables $Q_{u}^{(m)}$ referring to the power allocation per cluster. Let us define for each cluster $m$ the following useful parameters:

- $T_{m} \triangleq \sum_{n=1}^{N} A_{n, m}$ the number of sub-carriers in the cluster,

- $R_{u}^{(m)} \triangleq \sum_{\substack{n=1 \\ A_{n, m} \neq 0}}^{N} f_{s} \log _{2}\left(1+\frac{\gamma_{u}^{(m, n)}}{\Gamma_{u}}\right)$ the total achievable rate per cluster, 
- $\hat{Q}_{u}^{(m)} \triangleq \min _{\substack{1 \leq n \leq N \\ A_{n, m} \neq 0}}\left\{\frac{\hat{P}_{u}^{(n)}}{A_{n, m}}\right\}$ the maximum power per user per cluster.

These definitions allow the optimization problem (9) over the clusters of sub-carriers to be rewritten in an interesting form that only depends on the clusters (except for the interference constraint that we elaborate more in section IV-B):

$$
\begin{array}{ll} 
& \max _{Q_{u}^{(m)}} \sum_{u=1}^{K_{c}} \alpha_{u} \sum_{m=1}^{M} R_{u}^{(m)} \\
\text { S.t. } & \sum_{m=1}^{M} T_{m} Q_{u}^{(m)} \leq \hat{P}_{u} \forall u \\
& 0 \leq Q_{u}^{(m)} \leq \hat{Q}_{u}^{(m)} \forall u, \forall m \\
& A_{n, m} B_{l}^{(n)} \sum_{u=1}^{K_{c}}\left|H_{p_{l}, c_{u}}^{(n)}\right|^{2} Q_{u}^{(m)} \leq \check{I}_{l}^{(n)} \forall l, \forall(m, n) .
\end{array}
$$

By setting $M=N$, the interpolation matrix is the identity matrix $\left(\mathbf{A}=\mathbf{I}_{N \times N}\right)$ and the problem (10), is exactly the original power allocation problem without clustering.

\section{B. Per-cluster Sub-problems Decomposition}

The problem (10) is a non-convex optimization problem due to the co-channel interference between users. A common way to solve this primal problem is to derive and solve its equivalent dual problem by moving the constraints to the objective using a Lagrange multiplier per constraint. For nonconvex problems, there is a duality gap; however [8] has shown that for non convex optimization problems the duality gap is 0 under a time-sharing condition. The latter is typically satisfied for practical multiuser spectrum optimization problems in multicarrier systems when the number of sub-carriers is sufficiently high or goes to infinity. Thus, reformulating the problem into its Lagrangian dual, we obtain

$$
\min _{\lambda \geq 0}\left\{\begin{array}{l}
\max _{Q_{u}^{(m)}} \sum_{u=1}^{K_{c}}\left\{\alpha_{u} R_{u}+\lambda_{u}\left(\hat{P}_{u}-P_{u}\right)\right\} \\
\text { S.t } 0 \leq Q_{u}^{(m)} \leq \hat{Q}_{u}^{(m)} \forall u, \forall m \\
A_{n, m} B_{l}^{(n)} \sum_{u=1}^{K_{c}}\left|H_{p_{l}, c_{u}}^{(n)}\right|^{2} Q_{u}^{(m)} \leq \check{I}_{l}^{(n)} \forall l, \forall(m, n) .
\end{array}\right\}
$$

where $\boldsymbol{\lambda}=\left[\lambda_{1}, \ldots, \lambda_{K_{c}}\right]^{T}$ are the Lagrangian parameters. The problem (11) can be rewritten as

$$
\min _{\lambda \geq 0}\left[g(\boldsymbol{\lambda})+\sum_{u} \lambda_{u} \hat{P}_{u}\right],
$$

where the sub-problem $g(\boldsymbol{\lambda})$ is defined by

$$
\begin{gathered}
g(\boldsymbol{\lambda})=\max _{Q_{u}^{(m)}} \sum_{u=1}^{K_{c}}\left(\alpha_{u} \sum_{m=1}^{M} R_{u}^{(m)}-\lambda_{u} \sum_{m=1}^{M} T_{m} Q_{u}^{(m)}\right) \\
\text { S.t } \quad 0 \leq Q_{u}^{(m)} \leq \hat{Q}_{u}^{(m)} \forall u \\
A_{n, m} B_{l}^{(n)} \sum_{u=1}^{K_{c}}\left|H_{p_{l}, c_{u}}^{(n)}\right|^{2} Q_{u}^{(m)} \leq \check{I}_{l}^{(n)} \forall l, \forall n,
\end{gathered}
$$

which can be decomposed into sum of sub-problems over the each cluster of sub-carriers:

$$
g(\lambda)=\sum_{m=1}^{M} g^{(m)}(\lambda)
$$

where the sub-problem $g^{(m)}(\lambda)$ is defined by

$$
\begin{gathered}
g^{(m)}(\lambda)=\max _{Q_{u}^{(m)}} \sum_{u=1}^{K_{c}}\left(\alpha_{u} R_{u}^{(m)}-\lambda_{u} T_{m} Q_{u}^{(m)}\right) \\
\text { S.t } \quad 0 \leq Q_{u}^{(m)} \leq \hat{Q}_{u}^{(m)} \forall u \\
\quad A_{n, m} B_{l}^{(n)} \sum_{u=1}^{K_{c}}\left|H_{p_{l}, c_{u}}^{(n)}\right|^{2} Q_{u}^{(m)} \leq \check{I}_{l}^{(n)} \forall l, \forall n .
\end{gathered}
$$

Each of the sub-problems $g^{(m)}(\boldsymbol{\lambda})$ is a non-convex optimization problem in $K_{c}$ variables. These sub-problems can be solved optimally by exhaustive search over the discrete set of feasible power values. Clearly, this approach is not convenient for large users due to the exponential number of possible combinations. An alternative approach is to iterate over the users and optimize at each iteration the power for one user given the other users powers. This procedure has been shown to converge to a near-optimal solution of the multidimensional search [8], [9] with reduction of the computational cost from an exponential of the number of users $p^{K_{c}}$ to only $p \times K_{c}$ where $p$ is the number of power levels per user. The set of feasible powers is obtained by restricting the set of all affordable power levels (i.e., the powers corresponding to discrete bit-loading) for all users to only those satisfying the maximum power per cluster and the interference constraints to the primary users.

For the Lagrangian parameters $\{\boldsymbol{\lambda}\}$, an adaptive step-size search algorithm will be applied to determine their optimal values. Overall, the algorithm will consist of two main loops. In the outer loop a search over the feasible Lagrangian parameters using e.g. a sub-gradient decent method is performed. In an inner loop for each set of Lagrangian parameters, the power allocation per sub-carrier is optimized for each user.

The interesting part of this approach is that we show that the problem can be solved by transforming it into $M$ separate sub-problems over each cluster of sub-carriers. The clustering allows reducing the complexity by a factor equal to the number of sub-carriers per cluster $\frac{N}{M}$ thanks to the decoupling between the clusters. This decoupling allows writing the overall problem into a sum of elementary sub-problems that can be optimized separately.. The performance loss will depend on the spectral characteristics of the channels and the choice of the interpolation matrix $\mathbf{A}$ and especially on the number of sub-carriers per cluster $\frac{N}{M}$.

Interference Constraint per Cluster: We note that the interference constraints remain per sub-carrier due to the different interference channels for each user $\left|H_{p_{l}, c_{u}}^{(n)}\right|^{2}$. These constraints do not cause a harmful increase of the algorithm complexity since we are using them to only check if a set 
of power levels per cluster $\left(\left\{Q_{u}^{(m)}\right\}_{1 \leq u \leq K_{c}}\right)$ is feasible or not. Thus, we will have for a given possible power level of the cluster, $K_{p} \cdot T_{m}$ constraints to check (number of primary users multiplied by number of sub-carriers in the cluster $m$ ). If at least one of these $K_{p} \cdot T_{m}$ constraints is not fulfilled, the power level is discarded from the search space. Ideally, these $K_{p} \cdot T_{m}$ constraints per cluster could be transformed into $K_{p}$ constraints having the following format

$$
B_{l}^{(m)} \sum_{u=1}^{K_{c}}\left|\tilde{H}_{p_{l}, c_{u}}^{(m)}\right|^{2} Q_{u}^{(m)} \leq \tilde{I}_{l}^{(m)} \quad \forall l, \forall m,
$$

where $\tilde{I}_{l}^{(m)}$ and $\tilde{H}_{p_{l}, c_{u}}^{(m)}$ are respectively the equivalent interference threshold of the $l$-th primary user over the cluster $m$ and the equivalent channel gain from the $u$-th cognitive user towards the $l$-th primary user over the cluster $m$. Although, the exact expressions of $\tilde{I}_{l}^{(m)}$ and $\tilde{H}_{p_{l}, c_{u}}^{(m)}$ do not exist due to the multipath between cognitive users towards primary users, modified constraints can be used. For example, we allow the interference to exceed the interference level for a given sub-carrier but the average interference over the cluster should not exceed the average threshold levels of the same cluster. Mathematically speaking, this requirement result in the following expressions of the equivalent thresholds and channel gains as

$$
\begin{cases}\tilde{I}_{l}^{(m)}=\frac{1}{T_{m}} \sum_{\substack{n=1 \\ A_{n, m} \neq 0}}^{N} \check{I}_{l}^{(n)} & \forall l, \forall m \\ \left|\tilde{H}_{p_{l}, c_{u}}^{(m)}\right|^{2}=\frac{1}{T_{m}} \sum_{\substack{n=1 \\ A_{n, m} \neq 0}}^{N} A_{n, m}\left|H_{p_{l}, c_{u}}^{(n)}\right|^{2} & \forall u, \forall l, \forall m .\end{cases}
$$

Another example is to consider a worst-case interference threshold and interference gains per cluster enforcing the interference constraint for all sub-carriers are shown to be respectively

$$
\begin{cases}\tilde{I}_{l}^{(m)}=\min _{\substack{1 \leq n \leq N \\ A_{n, m} \neq 0}}\left\{\check{I}_{l}^{(n)}\right\} & \forall l, \forall m \\ \left|\tilde{H}_{p_{l}, c_{u}}^{(m)}\right|^{2}=\max _{\substack{1 \leq n \leq N \\ A_{n, m} \neq 0}}\left\{A_{n, m}\left|H_{p_{l}, c_{u}}^{(n)}\right|^{2}\right\} & \forall u, \forall l, \forall m .\end{cases}
$$

\section{Numerical Simulations}

For the numerical evaluation, we consider the primary and secondary users to be uniformly distributed in a cellular cell of radius one $\mathrm{Km}$. The channel gains, distributed as multivariate Rayleigh fading channels, are generated from multivariate complex Gaussian distribution with a covariance matrix as in [10]. The covariance between two sub-carriers is exponentially decaying as a function of the distance between the sub-carriers while the average power is proportional to the path-loss with a path-loss exponent $\eta=4$. The budget power is set to $20 \mathrm{dBm}$ per user while the maximum power per sub-carrier is $-2.3 \mathrm{dBm}$. We consider single primary user $\left(K_{p}=1\right)$. We assume the primary user present in $\nu=50 \%$ of the subcarriers. The interference threshold is computed such that the primary bit-loading in presence of cognitive users does not decrease by more than a degradation factor $\epsilon$ compared to its original bit loading in absence of cognitive user's interference. We run the simulations for a frequency band of $N=512$ subcarriers with different number of clusters $M$ and represent the results in function of the clustering factor $\rho=\frac{M}{N}$. We use the binary interpolation model $\left(A_{n, m}=0\right.$ or 1$)$ and equal number of sub-carriers per cluster.

In Fig. 1, we plot the achievable rate regions of two users while varying the number of clusters. We first note that the reduced dimension almost has no effect on the achievable rate even with $\rho=1 \%$ as compared to no clustering ( $\rho=100 \%$ ). This proves the efficiency of the reduced dimension approach since it allows to achieve a near-optimal performance with a much lower computational complexity that is proportional to the clustering factor as shown in section IV-B. The figure also shows the rate improvement for the cognitive users using the proposed hybrid scheme compared to the interweave or underlay modes due to the more opportunistic use of the available spectrum.

In Fig. 2, we draw the rate regions for different interference threshold levels by varying the factor $\epsilon$ which indicates the allowed reduction of the bit loading in each sub-carrier. We note that a factor $\epsilon=50 \%$ allows the secondary users to achieve almost their maximal performance (like without interference constraint). As the interference constraint become more strict (i.e., when $\epsilon$ decreases as in the $\epsilon=1 \%$ curve) the performance loss of the reduced dimension with respect to the full optimization increases which can be explained by the more strict constraint especially that this constraint should be respected for all sub-carriers in the cluster (if at least one subcarrier has a strong interference channel towards one of the primary users, then all sub-carriers in the same cluster should back-off to comply with the interference threshold).

In Fig. 3, we compare the performance when using an interference constraint per sub-carrier to the simplified interferences per cluster: the average interference (17) and the worst case interference (18). The received interference at the primary users are plotted in Fig. 4. The average interference constraint allows to achieve better rates but it violates the interference constraint for some carriers even-though the average interference in the cluster is the same. The worst case interference achieves approximately the same performance as the per carrier interference for this topology. With a higher number of users (primary and secondary), this interference is expected to give worse interference but for this case there is no effect due to the limited diversity of the channels.

\section{CONCLUSION}

In this paper, we proposed a reduced dimension resource allocation for cognitive radio by transforming the problem from an optimization over the sub-carriers to clusters of subcarriers. We proved that this approach allows the reduction 


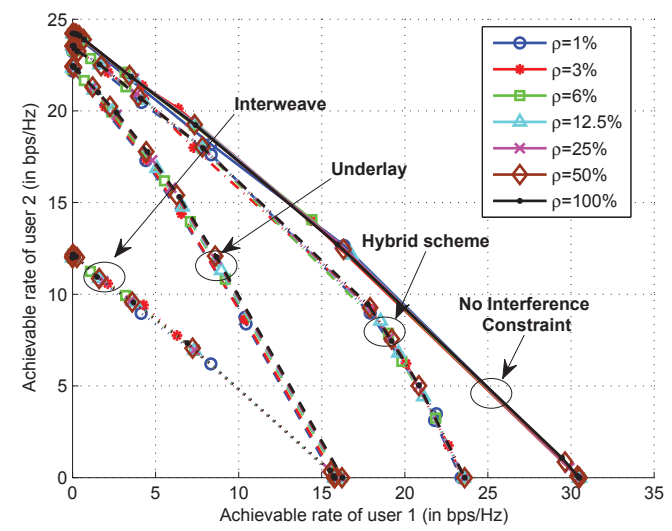

Fig. 1. Rate region of two users with reduced dimension power allocation using different clustering factor $\rho$ and an interference factor $\epsilon=10 \%$.

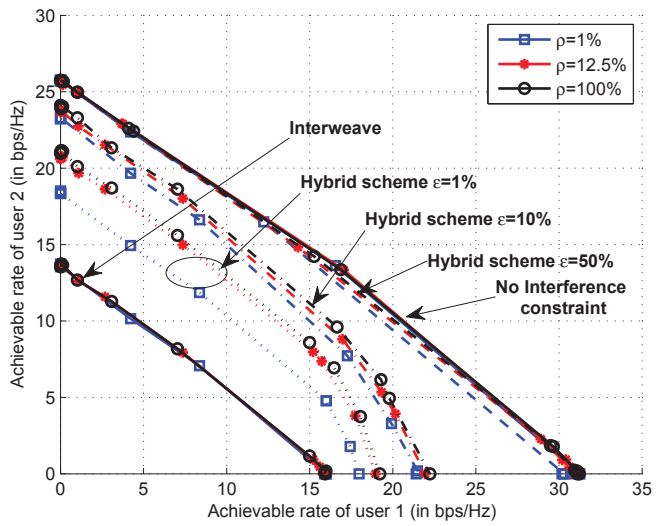

Fig. 2. Rate region of two users versus the clustering $\rho$ and the interference factors $\epsilon$.

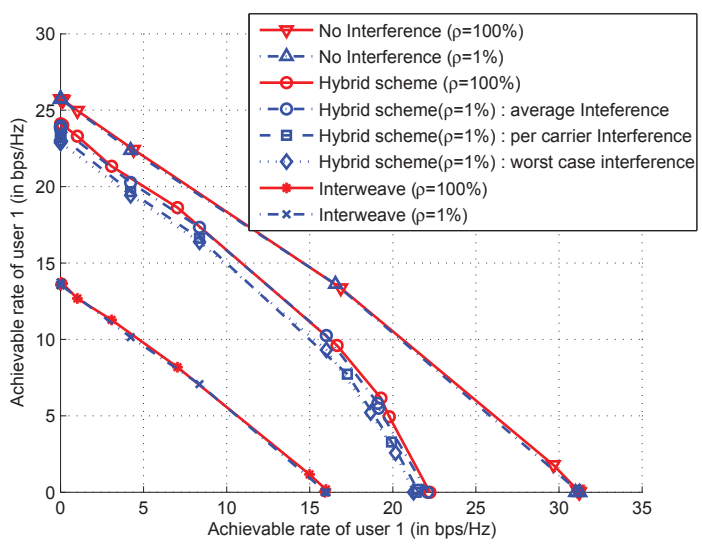

Fig. 3. Rate region of two users for different modified interference constraints per cluster.

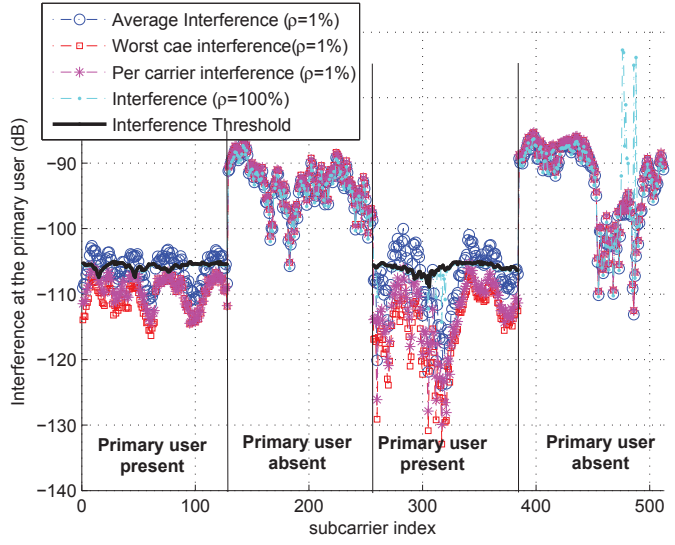

Fig. 4. Comparison of the Interference at the primary user with the different interference constraints with reduced dimension.

of the optimal resource allocation algorithm complexity by a factor equivalent to the number of sub-carriers per cluster while achieving close performance with comparison to that resulting from the optimization on all sub-carriers at least for the evaluated scenario. Obviously the performance gap between the proposed resource allocation and optimal solution will depend on the spectral properties of the channels and the different noises. Further researches are also under investigation to optimize the way sub-carriers are clustered in order to achieve highest performance with lower complexity.

\section{REFERENCES}

[1] J. Mitola III and G. Q. Macguire Jr., "Cognitive radio: Making software radios more personal", IEEE Personal Commun. Mag., vol. 6, no. 4, pp. 13-18, Aug. 1999.

[2] Federal Communications Commission: "Spectrum policy task force", Report ET Docket, Nov. 2002.

[3] A. Goldsmith; S.A. Jafar; I. Maric; S. Srinivasa; , "Breaking spectrum gridlock with cognitive radios: An information theoretic perspective", Proceedings of the IEEE, vol.97, no.5, pp.894-914, May 2009.

[4] F. Ian.; Akyildiz; Won-Yeol Lee; C. Mehmet; Vuran; Shantidev Mohanty; , "NeXt generation/dynamic spectrum access/cognitive radio wireless networks: A survey," Computer Networks, Volume 50, Issue 13, 15 September 2006, Pages 2127-2159.

[5] Seung-Jun Kim; G. B. Giannakis; , "Optimal resource allocation for MIMO ad hoc cognitive radio networks," in Proc. 46th Annual Allerton Conference on Communication, Control, and Computing, pp.39-45, Allerton House, IL, USA, Sep. 2008.

[6] Rui Zhang; Ying-Chang Liang; Shuguang Cui; , "Dynamic Resource Allocation in Cognitive Radio Networks," IEEE Signal Processing Magazine, vol.27, no.3, pp.102-114, May 2010.

[7] M. Guenach; C. Nuzman; K. Hooghe; J. Maes; M. Peeters; , "Reduced dimensional power optimization using class $A B$ and $G$ line drivers in DSL," in Proc. IEEE Global Communications Conference (GLOBECOM), pp.1443-1447, Miami, FL, USA, Dec. 2010.

[8] Wei Yu; R. Lui;, "Dual methods for nonconvex spectrum optimization of multicarrier systems," IEEE Transactions Communications, vol.54, no.7, pp.1310-1322, July 2006

[9] S. Huberman; C. Leung; T. Le-Ngoc; , "Dynamic spectrum management (DSM) algorithms for multi-user $x D S L "$, IEEE Communications Surveys \& Tutorials, vol.14, no.1, pp.109-130, First Quarter 2012.

[10] G. K. Karagiannidis; D. A. Zogas; S. A. Kotsopoulos; , "An efficient approach to multivariate Nakagami-m distribution using Green's matrix approximation", IEEE Transactions Wireless Communications, vol.2, no.5, pp. 883- 889, Sept. 2003 